\title{
Adaptive Synchronization for a Class of Cellular Neural Networks with Pantograph Delays
}

\author{
Xuehui Mei, ${ }^{1,2}$ Jiuju Xing, ${ }^{2}$ Haijun Jiang, ${ }^{2}$ Cheng Hu, ${ }^{2}$ and Liwei Zhang ${ }^{1}$ \\ ${ }^{1}$ College of Mathematics Sciences, Dalian University of Technology, Dalian 116024, China \\ ${ }^{2}$ College of Mathematics and System Sciences, Xinjiang University, Urumqi, Xinjiang 830046, China \\ Correspondence should be addressed to Haijun Jiang; jhj@xju.edu.cn
}

Received 15 July 2013; Accepted 7 November 2013

Academic Editor: Chun-Lei Tang

Copyright (c) 2013 Xuehui Mei et al. This is an open access article distributed under the Creative Commons Attribution License, which permits unrestricted use, distribution, and reproduction in any medium, provided the original work is properly cited.

\begin{abstract}
This paper deals with the synchronization problem for a class of cellular neural networks with pantograph delays. By using Lyapunov functional theory and inequality technique, some new and useful results are obtained for asymptotical synchronization under adaptive feedback controller.
\end{abstract}

\section{Introduction}

In recent several decades, since Chua and Yang $[1,2]$ proposed cellular neural networks in 1988, they have attracted considerable attention due to their potential applications in signal processing, image processing, and pattern recognition. So, the dynamical analysis of cellular neural networks is important and interesting from both theoretical and applied points of view; many effective research methods and important results have been presented in [3-13] and references cited therein.

Synchronization, as one of the most important collective behaviors, has attracted a wide range of research actively. Recently, synchronization of neural networks delays has been extensively studied in [11-29]. Several control schemes have been introduced to realize the network synchronization, for example, fuzzy control [25, 26], impulsive control [27, 29], intermittent control [11,28], and adaptive control [12, 13].

On the other hand, time delays are unavoidablely encountered in the signal transmission among the neurons due to the finite switching speed of neurons and amplifiers, which will affect the stability of the neural networks and may lead to some complex dynamic behaviors, such as instability, chaos, oscillation, and other performance of the neural network. Therefore, it is a very important research on the delayed neural networks. In view of the significance of the control for delayed neural networks, in recent years, there were many good results $[3,4,6-11,13,15-24,28,30-32]$. As we know, pantograph delays are playing more and more important role in some fields. For example, pantograph delay is usually required in Web Quality of Service (QoS) routing decision. However, few authors have taken care of neural networks with pantograph delays.

In [8], Ding studied the synchronization of delayed fuzzy cellular neural networks with impulses by using a nonimpulsive system to replace the impulsive system and some synchronization criteria were obtained by the well-known Lasalle invariant principle. In [11], the authors studied the lag synchronization for delayed fuzzy cellular neural networks via periodically intermittent control. The synchronization for a class of delayed fuzzy cellular neural networks with the parameters unknown was investigated (see [13]), by means of the Lasalle invariant principle of functional equations and the adaptive control method. In [30], the synchronization control of stochastic neural networks with time-varying delays is discussed by the Lyapunov functional method and linear inequality approach.

To the best of our knowledge, the adaptive synchronization results on neural networks with delays are usually based on Lasalle invariant principle, and there are few or even no results with regard to the adaptive synchronization for cellular neural networks with pantograph delays by applying adaptive feedback control. Based on the above analysis, in this paper, we will investigate the adaptive synchronization for cellular neural networks with pantograph delays by 
using Lyapunov functional theory, inequality technique, and Barbalat lemma, some new and useful criteria are derived.

This paper is organized as follows. In Section 2, model description and preliminaries are given. Some synchronization criteria are obtained in Section 3 under the adaptive feedback controller we assumed. In Section 4, the effectiveness and feasibility of the developed methods are shown by a numerical example.

\section{Preliminaries}

Motivated by the analysis in the above section, in this paper, we consider a class of cellular neural networks with pantograph delays, which is described by the following model:

$$
\begin{aligned}
\dot{x}_{i}(t)= & -a_{i} x_{i}(t)+\sum_{j=1}^{n} b_{i j} f_{j}\left(x_{j}(t)\right) \\
& +\sum_{j=1}^{n} c_{i j} f_{j}\left(x_{j}\left(q_{j} t\right)\right)+I_{i}(t), \quad i \in \mathcal{I},
\end{aligned}
$$

for $t>0$, where $i, j \in \mathscr{I}=\{1,2, \ldots, n\} ; x_{i}(t)$ denotes the state of the $i$ th neuron at the time $t ; a_{i}>0$ represents the rate with which the $i$ th neuron will reset its potential to the resting state in isolation when disconnected from the network and external input; $b_{i j}$ denotes the strength of the $j$ th neuron on the $i$ th neuron at the time $t ; c_{i j}$ denotes the strength of the $j$ th neuron on the $i$ th neuron at the time $q_{j} t ; f_{j}(\cdot)$ corresponds to the output of the $j$ th neuron; and $q_{j}(j \in$ $\mathscr{I})$ is constant and satisfies $0<q_{j}<1, q_{j} t=t-\left(1-q_{j}\right) t$, in which $\left(1-q_{j}\right) t$ denotes the pantograph delays along the axon of the $j$ th node and $I_{i}(t)$ corresponds to the external bias on the $i$ th neuron.

System (1) is supplemented with initial value given by

$$
x_{i}(0)=x_{i 0}, \quad i \in \mathscr{I} .
$$

In order to observe the synchronization behavior of system (1), the slaver system is designed as follows:

$$
\begin{aligned}
\dot{y}_{i}(t)= & -a_{i} y_{i}(t)+\sum_{j=1}^{n} b_{i j} f_{j}\left(y_{j}(t)\right) \\
& +\sum_{j=1}^{n} c_{i j} f_{j}\left(y_{j}\left(q_{j} t\right)\right)+I_{i}(t)+K_{i}(t), \quad i \in \mathscr{I},
\end{aligned}
$$

for $t>0$, where $y_{i}(t)$ denotes the state of the slave system, the rest of the notations are the same as in system (1), and $K_{i}(t)$ is a control input to be designed.

The initial value of system (3) is given by

$$
y_{i}(0)=y_{i 0}, \quad i \in \mathscr{I} \text {. }
$$

Assume that $x(t)=\left(x_{1}(t), \ldots, x_{n}(t)\right)^{T}$ and $y(t)=$ $\left(y_{1}(t), \ldots, y_{n}(t)\right)^{T}$ are solutions of systems (1) and (3) with different initial values $x_{i}(0)=x_{i 0}$ and $y_{i}(0)=y_{i 0}$ at the time $t=0$. Let $e_{i}(t)=y_{i}(t)-x_{i}(t)$ be the synchronization error between the states of the drive system (1) and response system (3); subtracting (1) from (3) yields the following error system:

$$
\begin{aligned}
\dot{e}_{i}(t)= & -a_{i} e_{i}(t)+\sum_{j=1}^{n} b_{i j}\left[f_{j}\left(y_{j}(t)\right)-f_{j}\left(x_{j}(t)\right)\right] \\
& +\sum_{j=1}^{n} c_{i j}\left[f_{j}\left(y_{j}\left(q_{j} t\right)\right)-f_{j}\left(x_{j}\left(q_{j} t\right)\right)\right]+K_{i}(t),
\end{aligned}
$$

$i \in \mathscr{I}$,

where the adaptive feedback controller $K_{i}(t)$ is designed by

$$
K_{i}(t)=\varepsilon_{i}(t) e_{i}(t)
$$

The feedback strength $\varepsilon(t)=\left(\varepsilon_{1}(t), \ldots, \varepsilon_{n}(t)\right)^{T}$ is adapted by the following law:

$$
\dot{\varepsilon}_{i}(t)=-\lambda_{i} e_{i}^{2}(t),
$$

where $\lambda_{i}>0(i=1,2, \ldots, n)$.

In order to further study systems (1) and (3) and obtain the main results, the following assumptions are necessary.

Assumption 1. We assume that there exist positive constants $M_{i}(i \in \mathscr{I})$ such that activation function $f_{i}(\cdot)$ satisfies the following condition:

$$
\left|f_{i}(x)\right| \leq M_{i}, \quad i \in \mathscr{I} .
$$

Assumption 2. For each $i \in \mathscr{I}$, we assume activation function $f_{i}(\cdot)$ satisfies the Lipschitz condition; that is, there exists constant $L_{i}$ such that

$$
\left|f_{i}(x)-f_{i}(y)\right| \leq L_{i}|x-y|, \quad \forall x, y \in R, i \in \mathscr{I} .
$$

On the synchronization of drive-response system (1) and (3), we have the following definition.

Definition 3. The master system (1) and the response system (3) are said to be synchronized, if for any solution $x(t)=$ $\left(x_{1}(t), \ldots, x_{n}(t)\right)^{T}$ of system (1) and any solution $y(t)=$ $\left(y_{1}(t), \ldots, y_{n}(t)\right)^{T}$ of system (3), we have

$$
\lim _{t \rightarrow \infty}\left(x_{i}(t)-y_{i}(t)\right)=0 \text {. }
$$

In addition, the following lemma is essential in establishing our main results.

Lemma 4 (Barbalat Lemma). Function $f(x)$ defined on interval $[0,+\infty)$; if $f(x)$ is uniformly continuous and $\int_{0}^{+\infty} f(x) d x<+\infty$ is satisfied. Then $\lim _{x \rightarrow+\infty} f(x)=0$.

\section{Adaptive Synchronization}

In this section, we will use Lyapunov functional theory, inequality technique, and Barbalat lemma to study the error system (5) realize globally asymptotical stable under adaptive feedback controller (6), that is, to realize the adaptive synchronization of drive system (1) and response system (3).

The following theorem is given to guarantee the synchronization of system (1) and (3). 
Theorem 5. Assume that Assumptions 1 and 2 hold, and the feedback strength $\varepsilon_{i}(t)$ is adapted by (7); then the error system (5) is globally asymptotical stable; that is, the drive system (1) and response system (3) are synchronized under adaptive feedback controller (6).

Proof. In order to establish the result of Theorem 5, we introduce the following Lyapunov functional:

$$
\begin{aligned}
V(t)= & \sum_{i=1}^{n}\left|e_{i}(t)\right|^{2}+\frac{1}{4} \sum_{i=1}^{n} \frac{1}{\lambda_{i}}\left(2 \varepsilon_{i}(t)+h\right)^{2} \\
& +\frac{1}{q_{i}} \sum_{i=1}^{n} \sum_{j=1}^{n}\left|c_{i j}\right| L_{j} \int_{q_{j} t}^{t} e_{j}^{2}(s) d s,
\end{aligned}
$$

where $h$ is a constant and $\underline{q}=\min _{i \in \mathcal{F}}\left\{q_{j}\right\}$, which will be given in the following.

From (5) and Assumption 2, we have

$$
\begin{aligned}
& D^{+}\left|e_{i}(t)\right| \\
& =\operatorname{sgn}\left(e_{i}(t)\right)\left\{-a_{i} e_{i}(t)\right. \\
& +\sum_{j=1}^{n} b_{i j}\left[f_{j}\left(y_{j}(t)\right)-f_{j}\left(x_{j}(t)\right)\right] \\
& +\sum_{j=1}^{n} c_{i j}\left[f_{j}\left(y_{j}\left(q_{j} t\right)\right)-f_{j}\left(x_{j}\left(q_{j} t\right)\right)\right] \\
& \left.+\varepsilon_{i}(t) e_{i}(t)\right\} \\
& \leq-a_{i}\left|e_{i}(t)\right|+\sum_{j=1}^{n}\left|b_{i j}\right|\left|f_{j}\left(y_{j}(t)\right)-f_{j}\left(x_{j}(t)\right)\right| \\
& +\sum_{j=1}^{n}\left|c_{i j}\right|\left|f_{j}\left(y_{j}\left(q_{j} t\right)\right)-f_{j}\left(x_{j}\left(q_{j} t\right)\right)\right|+\varepsilon_{i}(t)\left|e_{i}(t)\right| \\
& \leq\left(-a_{i}+\varepsilon_{i}(t)\right)\left|e_{i}(t)\right|+\sum_{j=1}^{n}\left|b_{i j}\right| L_{j}\left|e_{j}(t)\right| \\
& +\sum_{j=1}^{n}\left|c_{i j}\right| L_{j}\left|e_{j}\left(q_{j} t\right)\right|
\end{aligned}
$$

In the following, we calculate the upper right derivative of $V(t)$ along the solution of error system (5). From (11) and (12), we have

$D^{+} V(t)$

$$
=2 \sum_{i=1}^{n}\left|e_{i}(t)\right| D^{+}\left|e_{i}(t)\right|-\sum_{i=1}^{n}\left(2 \varepsilon_{i}(t)+h\right) e_{i}^{2}(t)
$$

$$
\begin{aligned}
& +\frac{1}{q_{i}} \sum_{i=1}^{n} \sum_{j=1}^{n}\left|c_{i j}\right| L_{j}\left[e_{j}^{2}(t)-q_{j} e_{j}^{2}\left(q_{j} t\right)\right] \\
& \leq 2 \sum_{i=1}^{n}\left|e_{i}(t)\right|\left\{\left(-a_{i}+\varepsilon_{i}(t)\right)\left|e_{i}(t)\right|+\sum_{j=1}^{n}\left|b_{i j}\right| L_{j}\left|e_{j}(t)\right|\right. \\
& \left.+\sum_{j=1}^{n}\left|c_{i j}\right| L_{j}\left|e_{j}\left(q_{j} t\right)\right|\right\} \\
& -2 \sum_{i=1}^{n} \varepsilon_{i}(t) e_{i}^{2}(t)-\sum_{i=1}^{n} h e_{i}^{2}(t) \\
& +\frac{1}{q} \sum_{i=1}^{n} \sum_{j=1}^{n}\left|c_{i j}\right| L_{j}\left[e_{j}^{2}(t)-q_{j} e_{j}^{2}\left(q_{j} t\right)\right] \\
& =2 \sum_{i=1}^{n}\left|e_{i}(t)\right|\left[-a_{i}\left|e_{i}(t)\right|+\sum_{j=1}^{n}\left|b_{i j}\right| L_{j}\left|e_{j}(t)\right|\right. \\
& \left.+\sum_{j=1}^{n}\left|c_{i j}\right| L_{j}\left|e_{j}\left(q_{j} t\right)\right|\right] \\
& -\sum_{i=1}^{n} h e_{i}^{2}(t)+\frac{1}{q_{i}} \sum_{i=1}^{n} \sum_{j=1}^{n}\left|c_{i j}\right| L_{j}\left[e_{j}^{2}(t)-q_{j} e_{j}^{2}\left(q_{j} t\right)\right] \\
& =-\sum_{i=1}^{n} 2 a_{i} e_{i}^{2}(t)+\sum_{i=1}^{n} \sum_{j=1}^{n} 2\left|b_{i j}\right| L_{j}\left|e_{i}(t)\right|\left|e_{j}(t)\right| \\
& +\sum_{i=1}^{n} \sum_{j=1}^{n} 2\left|c_{i j}\right| L_{j}\left|e_{i}(t)\right|\left|e_{j}\left(q_{j} t\right)\right| \\
& -\sum_{i=1}^{n} h e_{i}^{2}(t)+\frac{1}{q} \sum_{i=1}^{n} \sum_{j=1}^{n}\left|c_{i j}\right| L_{j}\left[e_{j}^{2}(t)-q_{j} e_{j}^{2}\left(q_{j} t\right)\right] \\
& \leq-\sum_{i=1}^{n} 2 a_{i} e_{i}^{2}(t)+\sum_{i=1}^{n} \sum_{j=1}^{n}\left|b_{i j}\right| L_{j}\left(e_{i}^{2}(t)+e_{j}^{2}(t)\right) \\
& +\sum_{i=1}^{n} \sum_{j=1}^{n}\left|c_{i j}\right| L_{j}\left(e_{i}^{2}(t)+e_{j}^{2}\left(q_{j} t\right)\right) \\
& -\sum_{i=1}^{n} h e_{i}^{2}(t)+\frac{1}{\underline{q}} \sum_{i=1}^{n} \sum_{j=1}^{n}\left|c_{i j}\right| L_{j}\left(e_{j}^{2}(t)-q_{j} e_{j}^{2}\left(q_{j} t\right)\right) \\
& =-\sum_{i=1}^{n}\left(2 a_{i}+h\right) e_{i}^{2}(t)+\sum_{i=1}^{n} \sum_{j=1}^{n}\left(\left|b_{i j}\right| L_{j}+\left|b_{j i}\right| L_{i}\right) e_{i}^{2}(t) \\
& +\sum_{i=1}^{n} \sum_{j=1}^{n}\left(\left|c_{i j}\right| L_{j}+\frac{1}{q}\left|c_{j i}\right| L_{i}\right) e_{i}^{2}(t) \\
& +\sum_{i=1}^{n} \sum_{j=1}^{n}\left|c_{i j}\right| L_{j}\left(1-\frac{q_{j}}{\underline{q}}\right) \\
& \leq-\sum_{i=1}^{n}\left(2 a_{i}+h\right) e_{i}^{2}(t)+\sum_{i=1}^{n} \sum_{j=1}^{n}\left(\left|b_{i j}\right| L_{j}+\left|b_{j i}\right| L_{i}\right) e_{i}^{2}(t)
\end{aligned}
$$




$$
\begin{gathered}
+\sum_{i=1}^{n} \sum_{j=1}^{n}\left(\left|c_{i j}\right| L_{j}+\frac{1}{q}\left|c_{j i}\right| L_{i}\right) e_{i}^{2}(t) \\
=-\sum_{i=1}^{n}\left[2 a_{i}+h-\sum_{j=1}^{n}\left(\left|b_{i j}\right| L_{j}+\left|b_{j i}\right| L_{i}\right)\right. \\
\left.\quad-\sum_{j=1}^{n}\left(\left|c_{i j}\right| L_{j}+\frac{1}{q}\left|c_{j i}\right| L_{i}\right)\right] e_{i}^{2}(t) .
\end{gathered}
$$

We choose

$$
\begin{aligned}
h= & \sum_{j=1}^{n}\left(\left|b_{i j}\right| L_{j}+\left|b_{j i}\right| L_{i}\right) \\
& +\sum_{j=1}^{n}\left(\left|c_{i j}\right| L_{j}+\frac{1}{\underline{q}}\left|c_{j i}\right| L_{i}\right)-2 a_{i}+1 .
\end{aligned}
$$

Then, we finally obtain

$$
D^{+} V(t) \leq-\sum_{i=1}^{n} e_{i}^{2}(t),
$$

For $t \geq 0$, integrating both sides of inequality (15) over $[0, t]$, we get

$$
V(t)-V(0) \leq-\int_{0}^{t} \sum_{i=1}^{n} e_{i}^{2}(s) d s .
$$

Then

$$
V(t)+\int_{0}^{t} \sum_{i=1}^{n} e_{i}^{2}(s) d s \leq V(0),
$$

In view of $V(0)>0$,

$$
\lim _{t \rightarrow \infty} \sup \int_{0}^{t} \sum_{i=1}^{n} e_{i}^{2}(s) d s \leq V(0)<+\infty .
$$

From (11) and (17), we have

$$
\sum_{i=1}^{n}\left|e_{i}(t)\right|^{2} \leq V(t) \leq V(0)
$$

Therefore, $e_{i}(t)$ is bounded on $[0,+\infty)$. From (12), we get that $\dot{e}_{i}(t)$ is bounded on $[0,+\infty)$. On the other hand, in view of (18), applying Lemma 4 , we have

$$
\lim _{t \rightarrow \infty} e_{i}(t)=0
$$

which implies that the error system (5) is globally asymptotically stable; that is, the drive-response systems (1) and (3) are synchronized under adaptive feedback controller (6). The proof of Theorem 5 is completed.

The following results are easily obtained from Theorem 5.
Corollary 6. Under Assumptions 1 and 2, the masterresponse system (1) and (3) can be synchronized under the adaptive feedback controller (6) and (7), if there exists a constant $h$ satisfying the following condition.

Assumption 7. $\sum_{j=1}^{n}\left(\left|b_{i j}\right| L_{j}+\left|b_{j i}\right| L_{i}\right)+\sum_{j=1}^{n}\left(\left|c_{i j}\right| L_{j}+\right.$ $\left.(1 / \underline{q})\left|c_{j i}\right| L_{i}\right)-2 a_{i}-h<0$, where $\underline{q}=\min _{i \in \mathcal{I}}\left\{q_{i}\right\}$.

In particular, when $q_{j}=q(0<q<1)$, the masterresponse systems (1) and (3) have the following special case:

$$
\begin{aligned}
\dot{x}_{i}(t)= & -a_{i} x_{i}(t)+\sum_{j=1}^{n} b_{i j} f_{j}\left(x_{j}(t)\right) \\
& +\sum_{j=1}^{n} c_{i j} f_{j}\left(x_{j}(q t)\right)+I_{i}(t), \quad i \in \mathscr{I}, \\
\dot{y}_{i}(t)= & -a_{i} y_{i}(t)+\sum_{j=1}^{n} b_{i j} f_{j}\left(y_{j}(t)\right) \\
& +\sum_{j=1}^{n} c_{i j} f_{j}\left(y_{j}(q t)\right)+I_{i}(t)+K_{i}(t), \quad i \in \mathscr{I} .
\end{aligned}
$$

From Theorem 5, we have the following corollary as a special case of Theorem 5 .

Corollary 8. Assume Assumptions 1 and 2 hold, the master system (21) and response system (22) can be adaptively synchronized under the adaptive feedback controller (6) and (7), if there exists a constant $h$ satisfying the following condition.

Assumption 9. $\sum_{j=1}^{n}\left(\left|b_{i j}\right| L_{j}+\left|b_{j i}\right| L_{i}\right)+\sum_{j=1}^{n}\left(\left|c_{i j}\right| L_{j}+\right.$ $\left.(1 / q)\left|c_{j i}\right| L_{i}\right)-2 a_{i}-h<0$.

If the feedback strength $\varepsilon(t)=(\alpha(t), \ldots, \alpha(t))^{T}$, then the response system (3) has the special case as follows:

$$
\begin{aligned}
\dot{y}_{i}(t)= & -a_{i} y_{i}(t)+\sum_{j=1}^{n} b_{i j} f_{j}\left(y_{j}(t)\right) \\
& +\sum_{j=1}^{n} c_{i j} f_{j}\left(y_{j}\left(q_{j} t\right)\right)+I_{i}(t)+\alpha(t) e_{i}(t), \quad i \in \mathscr{I} .
\end{aligned}
$$

Thus, we obtain the following corollary as another special case of Theorem 5 .

Corollary 10. Under Assumptions 1 and 2, the masterresponse system (1) and (23) can be synchronized under the adaptive feedback controller (6), if there exists a constant $\lambda>$ 0 such that feedback strength $\alpha(t)$ is adapted duly according to the following updated law:

$$
\dot{\alpha}(t)=-\lambda e_{i}^{2}(t) .
$$

Remark 11. In [13], based on Lasalle invariant principle of functional differential equations and the adaptive feedback control technique, the adaptive synchronization behavior for the delayed fuzzy cellular neural networks was obtained. In 
this paper, by applying Lyapunov functional theory, inequality technique, and Barbalat lemma, some useful results are derived for asymptotical synchronization under adaptive feedback controller.

Remark 12. Recently, research on synchronization problem for chaotic systems, authors have considered constant delay, time-varying delays, distributed delays, mixed time-varying delays, and so on. However, to the best of our knowledge, there are few results concerning pantograph delays for chaotic systems. So, in this paper, the adaptive synchronization of a class of cellular neural networks with pantograph delays was derived.

Remark 13. As far as we know, the synchronization of chaotic systems has been intensively investigated and many good results have been obtained by using control schemes such as adaptive control, pinning control, fuzzy control, impulsive control, intermittent control, and so on. However, to the best of our knowledge, there are few results concerning the synchronization for a class of unautonomous cellular neural networks with pantograph delays based on intermittent feedback control. Therefore, this is another interesting open problem that we should study in the future.

\section{Numerical Simulations}

In this section, one chaotic network is given to show the effectiveness of our results obtained in this paper.

Example 1. Consider the following cellular neural networks with pantograph delays described by

$$
\begin{aligned}
\dot{x}_{i}(t)= & -a_{i} x_{i}(t)+\sum_{j=1}^{n} b_{i j} f_{j}\left(x_{j}(t)\right) \\
& +\sum_{j=1}^{n} c_{i j} f_{j}\left(x_{j}\left(q_{j} t\right)\right)+I_{i}(t), \quad i=1,2,
\end{aligned}
$$

where $i=1,2, f_{1}(x)=f_{2}(x)=(1 / 2)(|x+1|-|x-1|)$, $a_{1}=a_{2}=1, b_{11}=2.3, b_{12}=3.0, b_{21}=-5.0, b_{22}=-0.1$, $c_{11}=-1.5, c_{12}=0.1, c_{21}=1.0, c_{22}=2.4$, and $q_{1}=q_{2}=0.1$.

The numerical simulation of system (25) is represented in Figure 1, which shows that system (25) has a chaotic attractor.

In the following, we consider the adaptive synchronization of drive system (25) and response system described by

$$
\begin{aligned}
\dot{y}_{i}(t)= & -a_{i} y_{i}(t)+\sum_{j=1}^{n} b_{i j} f_{j}\left(y_{j}(t)\right) \\
& +\sum_{j=1}^{n} c_{i j} f_{j}\left(y_{j}\left(q_{j} t\right)\right)+I_{i}(t)+\varepsilon_{i}(t) e_{i}(t), \quad i=1,2,
\end{aligned}
$$

where $i=1,2$; the parameters $a_{i}, b_{i j}$, and $c_{i j}$ are defined as in system (25); and

$$
\dot{\varepsilon}_{i}(t)=-\lambda_{i} e_{i}^{2}(t), \quad i=1,2 .
$$



FIgURE 1: The chaotic behavior of system (25) with the initial values $x_{1}(s)=0.3, x_{2}(s)=-0.5$, for $s \in[-1,0]$.

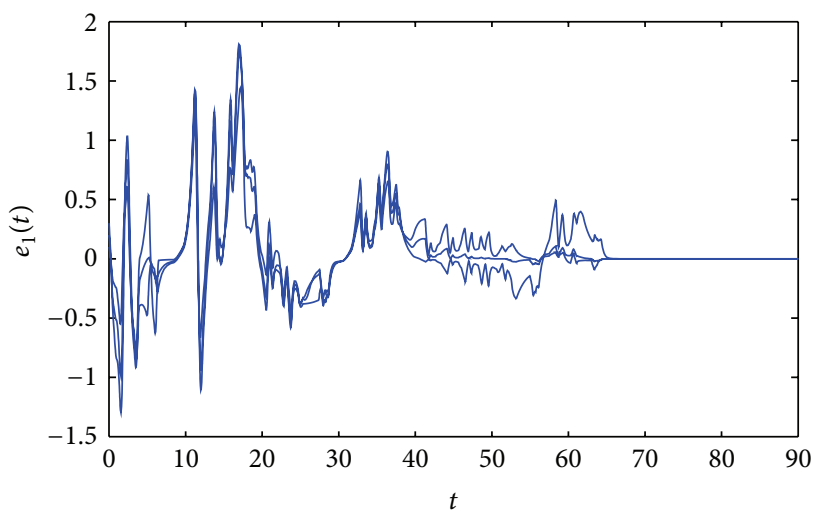

Figure 2: Synchronization errors of system (25) and system (26).

We choose the following initial condition associated with response system (26):

$$
\begin{gathered}
y_{1}(s)=0.5-0.1 k, \quad y_{2}(s)=-0.7+0.1 k, \\
k=-1,0,1, \quad \forall s \in[-1,0] .
\end{gathered}
$$

Furthermore, we choose in error system (27) the initial conditions $\varepsilon_{i}(0)=0(i=1,2)$ and $\lambda_{1}=0.3, \lambda_{2}=0.2$. By numerical simulation, we can see that the simulation results of master system (25) synchronize with response system (26) as shown in Figures 2, 3, 4, and 5.

\section{Conclusions}

In this paper, an adaptive controller has been proposed to investigate the adaptive synchronization for a class of cellular neural networks with pantograph delays by utilizing Lyapunov functional theory, inequality technique, and Barbalat lemma; some sufficient and useful conditions have been derived. Our synchronization criteria are easily verified and do not apply linear matrix inequality and the traditional Lasalle invariant principle. Finally, an example is given to verify the effectiveness and feasibility of the developed methods. 




FIGURE 3: Synchronization errors of system (25) and system (27).

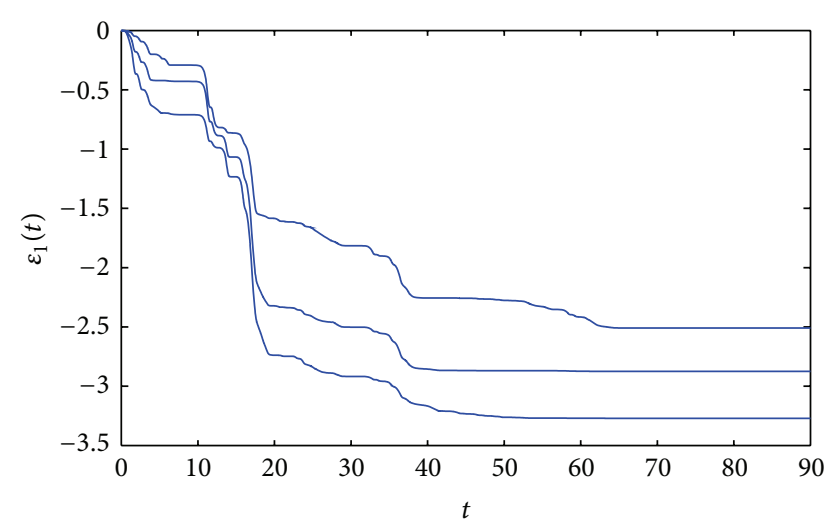

Figure 4: The curve of $\varepsilon_{1}(t)$.

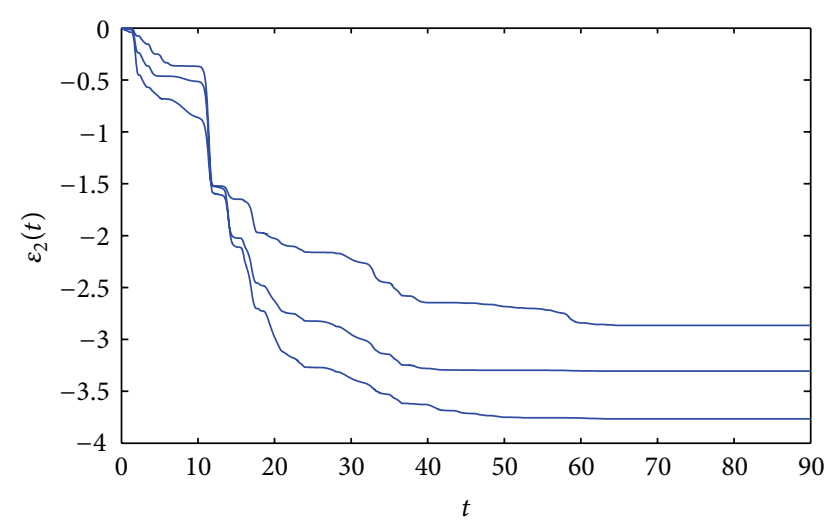

Figure 5: The curve of $\varepsilon_{2}(t)$.

\section{Acknowledgments}

This work was supported by Natural Science Foundation of Xinjiang (Grant No. 2013211B06), China Postdoctoral Science Foundation funded project (2013M540782), Natural Science Foundation of Xinjiang University (Grant No. BS120101), and National Natural Science Foundation of People's Republic of China (Grant No. 61164004).

\section{References}

[1] L. O. Chua and L. Yang, "Cellular neural networks: theory," IEEE Transactions on Circuits and Systems, vol. 35, no. 10, pp. 1257$1272,1988$.

[2] L. O. Chua and L. Yang, "Cellular neural networks: applications," IEEE Transactions on Circuits and Systems, vol. 35, no. 10, pp. 1273-1290, 1988.

[3] Z. J. Gui and X. S. Yang, "Stability and existence of periodic solutions of periodic cellular neural networks with time-varying delays," Computers \& Mathematics with Applications, vol. 52, no. 12, pp. 1657-1670, 2006.

[4] H. Jiang, Z. Li, and Z. Teng, "Boundedness and stability for nonautonomous cellular neural networks with delay," Physics Letters A, vol. 306, no. 5-6, pp. 313-325, 2003.

[5] Z. J. Gui and W. G. Ge, "Existence and uniqueness of periodic solutions of nonautonomous cellural neural networks with impulses," Physics Letters A, vol. 354, pp. 84-94, 2006.

[6] H. J. Jiang and Z. D. Teng, "A new criterion on the global exponential stability for cellural neural networks with multiple time-varying delays," Physics Letters A, vol. 338, pp. 461-471, 2005.

[7] Q. Song and J. Cao, "Dynamical behaviors of discrete-time fuzzy cellular neural networks with variable delays and impulses," Journal of the Franklin Institute, vol. 345, no. 1, pp. 39-59, 2008.

[8] W. Ding, "Synchronization of delayed fuzzy cellular neural networks with impulsive effects," Communications in Nonlinear Science and Numerical Simulation, vol. 14, no. 11, pp. 3945-3952, 2009.

[9] W. Ding, M. Han, and M. Li, "Exponential lag synchronization of delayed fuzzy cellular neural networks with impulses," Physics Letters A, vol. 373, no. 8-9, pp. 832-837, 2009.

[10] X. Gao, S. Zhong, and F. Gao, "Exponential synchronization of neural networks with time-varying delays," Nonlinear Analysis, vol. 71, no. 5-6, pp. 2003-2011, 2009.

[11] J. Yu, C. Hu, H. Jiang, and Z. Teng, "Exponential lag synchronization for delayed fuzzy cellular neural networks via periodically intermittent control," Mathematics and Computers in Simulation, vol. 82, no. 5, pp. 895-908, 2012.

[12] M. Hu and $\mathrm{Z}$. Xu, "Adaptive feedback controller for projective synchronization," Nonlinear Analysis: Real World Applications, vol. 9, no. 3, pp. 1253-1260, 2008.

[13] W. Ding and M. Han, "Synchronization of delayed fuzzy cellular neural networks based on adaptive control," Physics Letters A, vol. 372, no. 26, pp. 4674-4681, 2008.

[14] X. Yang and J. Cao, "Stochastic synchronization of coupled neural networks with intermittent control," Physics Letters A, vol. 373, no. 36, pp. 3259-3272, 2009.

[15] Y. Yang and J. Cao, "Exponential lag synchronization of a class of chaotic delayed neural networks with impulsive effects," Physica A, vol. 386, no. 1, pp. 492-502, 2007.

[16] X. Yang, J. Cao, Y. Long, and R. Wei, "Adaptive lag synchronization for competitive neural networks with mixed delays and uncertain hybrid perturbations," IEEE Transactions on Neural Networks, vol. 21, no. 10, pp. 1656-1667, 2010.

[17] X. Yang, C. Huang, and J. Cao, "An LMI approach for exponential synchronization of switched stochastic competitive neural networks with mixed delays," Neural Computing and Applications, vol. 21, no. 8, pp. 2033-2047, 2012.

[18] J. Cao and J. Lu, "Adaptive synchronization of neural networks with or without time-varying delay," Chaos, vol. 16, no. 1, Article ID 013133, 2006. 
[19] X. Huang and J. Cao, "Generalized synchronization for delayed chaotic neural networks: a novel coupling scheme," Nonlinearity, vol. 19, no. 12, pp. 2797-2811, 2006.

[20] P. Li, J. Cao, and Z. Wang, "Robust impulsive synchronization of coupled delayed neural networks with uncertainties," Physica A, vol. 373, pp. 261-272, 2007.

[21] Y. Sun, J. Cao, and Z. Wang, "Exponential synchronization of stochastic perturbed chaotic delayed neural networks," Neurocomputing, vol. 70, no. 13-15, pp. 2477-2485, 2007.

[22] Y. Sun and J. Cao, "Adaptive lag synchronization of unknown chaotic delayed neural networks with noise perturbation," Physics Letters A, vol. 364, no. 3-4, pp. 277-285, 2007.

[23] Q. Gan, R. Hu, and Y. Liang, "Adaptive synchronization for stochastic competitive neural networks with mixed timevarying delays," Communications in Nonlinear Science and Numerical Simulation, vol. 17, no. 9, pp. 3708-3718, 2012.

[24] Y. Tang, R. Qiu, J. Fang, Q. Miao, and M. Xia, "Adaptive lag synchronization in unknown stochastic chaotic neural networks with discrete and distributed time-varying delays," Physics Letters A, vol. 372, no. 24, pp. 4425-4433, 2008.

[25] C. Hu, H. Jiang, and Z. Teng, "Fuzzy impulsive control and synchronization of general chaotic system," Acta Applicandae Mathematicae, vol. 109, no. 2, pp. 463-485, 2010.

[26] K. Tanaka and H. O. Wang, "Fuzzy control of chaotic systems using LMIs: regulation, synchronization and chaos model following," in Proceedings of the IEEE International Conference on Fuzzy Systems, vol. 1, pp. 434-439, May 1998.

[27] H. Zhang, Z.-H. Guan, and D. W. C. Ho, "On synchronization of hybrid switching and impulsive networks," in Proceedings of the 45th IEEE Conference on Decision and Control (CDC '06), vol. 1, pp. 2765-2770, San Diego, Calif, USA, December 2006.

[28] W. Xia and J. Cao, "Pinning synchronization of delayed dynamical networks via periodically intermittent control," Chaos, vol. 19, no. 1, Article ID 013120, 2009.

[29] B. Liu, X. Liu, G. Chen, and H. Wang, "Robust impulsive synchronization of uncertain dynamical networks," IEEE Transactions on Circuits and Systems I, vol. 52, no. 7, pp. 1431-1441, 2005.

[30] W. Yu and J. Cao, "Synchronization control of stochastic delayed neural networks," Physica A, vol. 373, pp. 252-260, 2007.

[31] X. Liao, K. W. Wong, and Z. Wu, "Bifurcation analysis on a twoneuron system with distributed delays," Physica D, vol. 149, no. 1-2, pp. 123-141, 2001.

[32] J. Cao and D. W. C. Ho, "A general framework for global asymptotic stability analysis of delayed neural networks based on LMI approach," Chaos, Solitons \& Fractals, vol. 24, no. 5, pp. 1317-1329, 2005. 


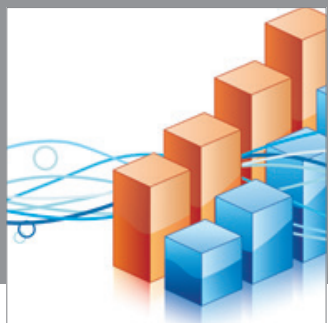

Advances in

Operations Research

mansans



The Scientific World Journal
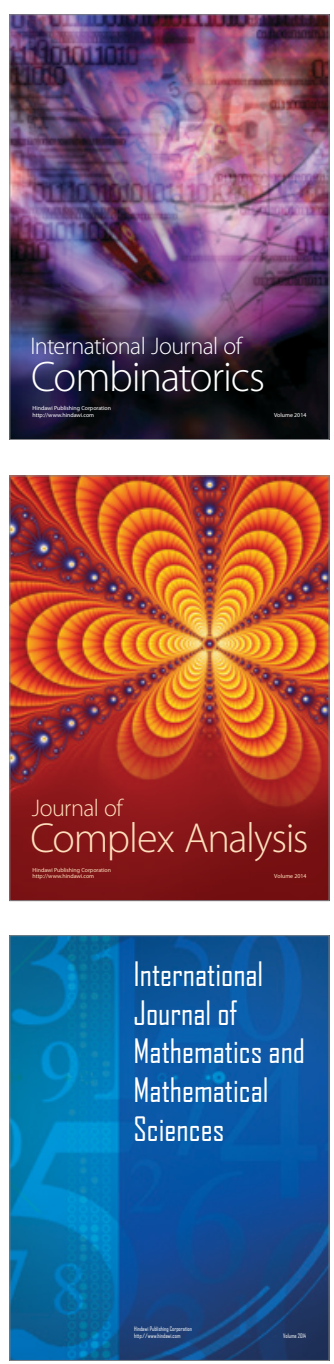
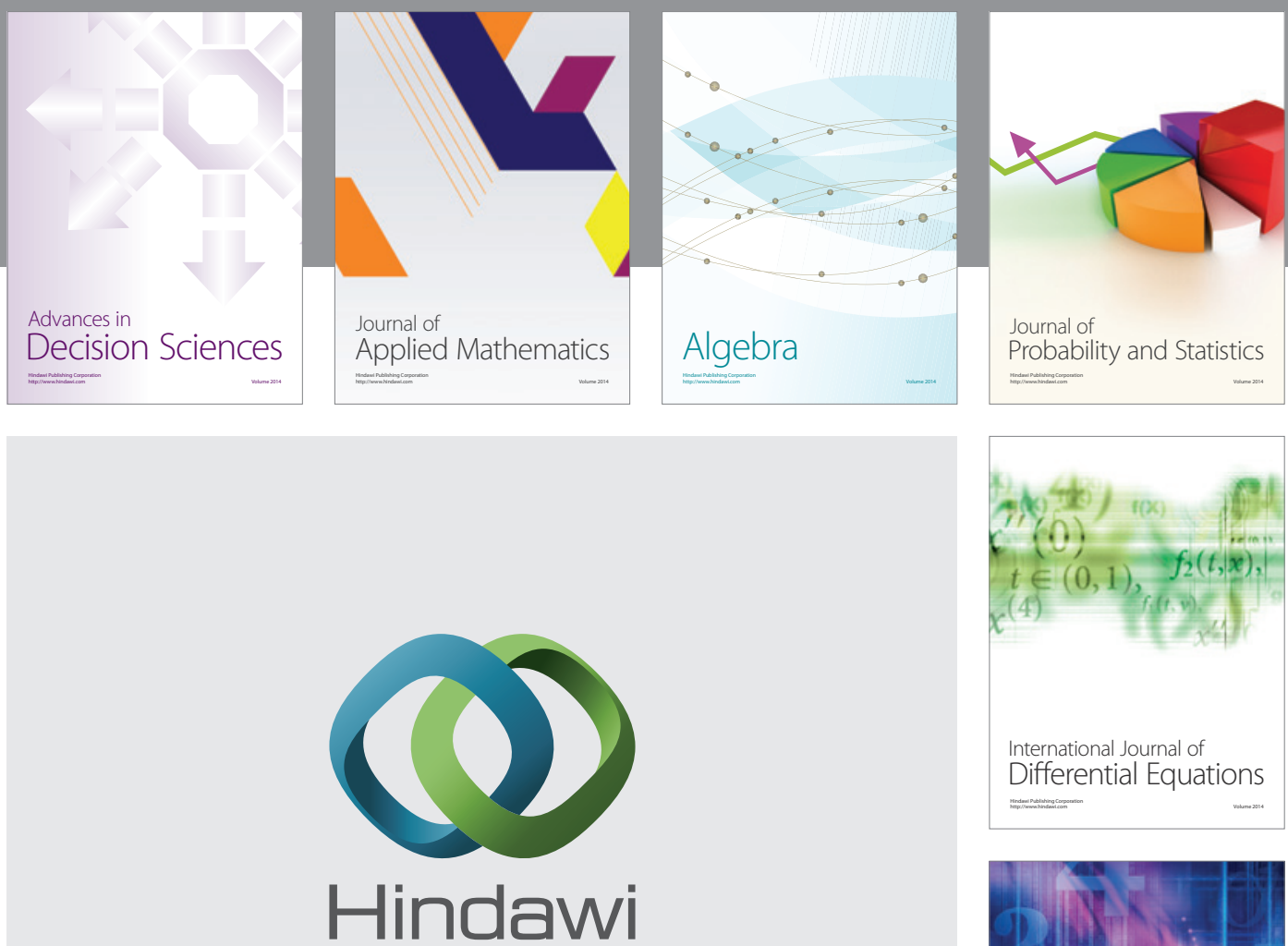

Submit your manuscripts at http://www.hindawi.com
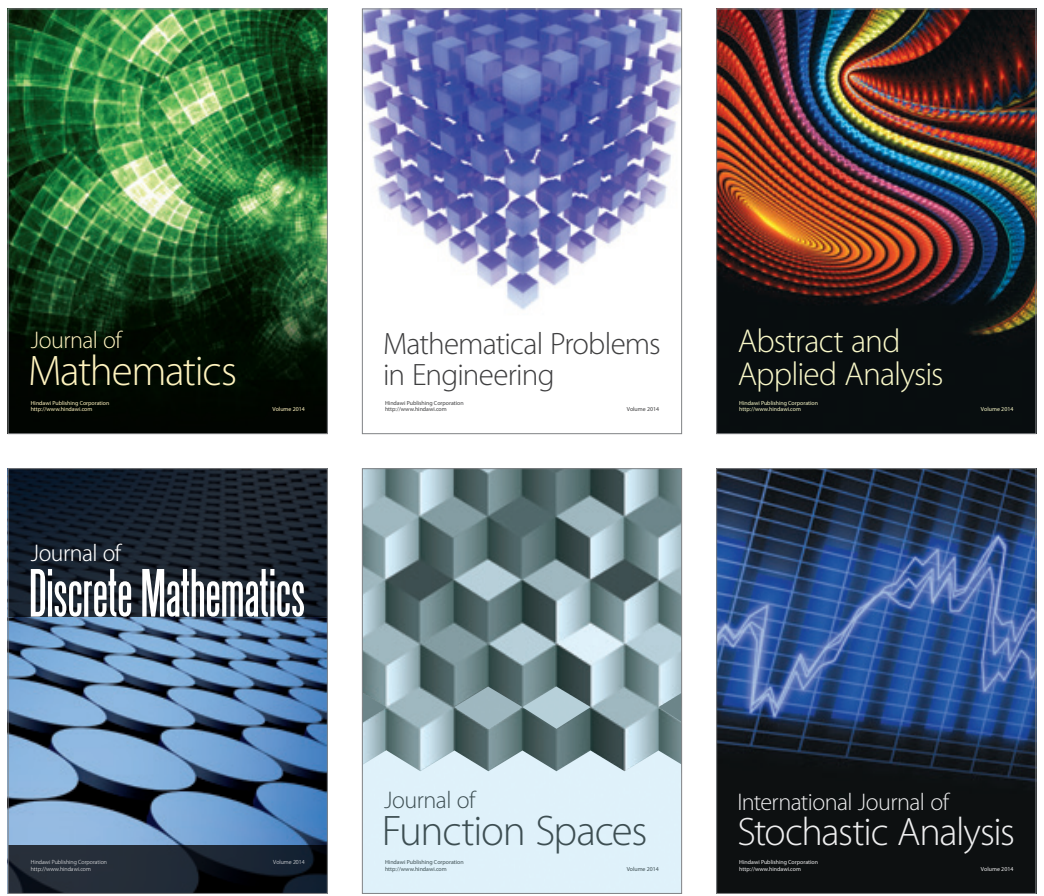

Journal of

Function Spaces



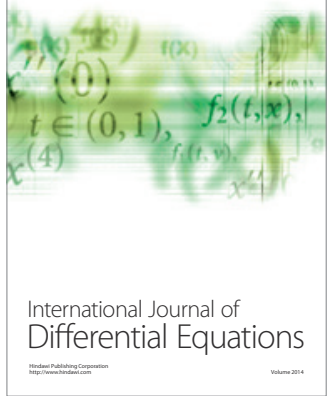
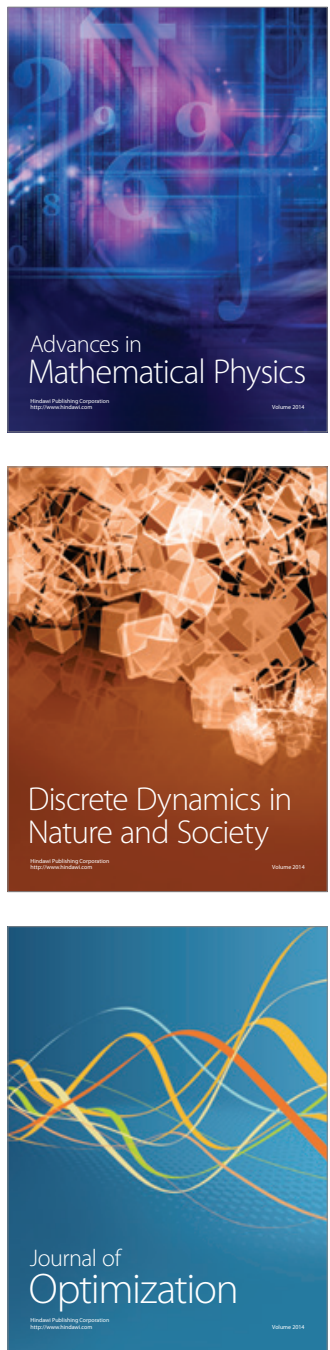